\title{
Higher cerebral function and hemispheric blood flow during awake carotid artery balloon test occlusions
}

R S Marshall, R M Lazar, J P Mohr, J Pile-Spellman, L Hacein-Bey, D H Duong, S Joshi, $\mathrm{X}$ Chen, Bruce Levin, W L Young

Department of

Neurology

R S Marshall

R M Lazar

J P Mohr

Department of Neurological Surgery

R M Lazar

J P Mohr

J Pile-Spellman

W L Young

Department of

Radiology

J Pile-Spellman

L Hacein-Bey

D H Duong

W L Young

Department of Biostatistics

$\mathrm{X}$ Chen

B Levin

Department of Anesthesiology, Columbia-Presbyterian

Medical Center,

New York

S Joshi

W L Young

Correspondence to: Dr Randolph S Marshall The Neurological Institute, Columbia-Presbyterian Medical Center, $710 \mathrm{~W}$ 168th Street, New York, NY 10032, USA.

Received 8 September 1998 and in revised form

7 December 1998

Accepted 24 December 1998

\begin{abstract}
Objectives-Measures capable of detecting early alterations of brain function during acute cerebral ischaemia have not been adequately defined. Internal carotid artery (ICA) test occlusions provide a well controlled environment to investigate the effects of reversible acute hemispheric hypoperfusion on brain function. A continuous time estimation task was developed that reliably demonstrated alterations in higher cerebral function before development of any deficits in elementary neurological function during ICA balloon catheter test occlusions. In the present study, we hypothesised that alterations in our behavioural task would correlate significantly with absolute level of ipsilateral CBF.
\end{abstract}

Methods-Twenty five patients underwent awake ICA test occlusions during which sustained attention and ipsilateral CBF were measured simultaneously using our time estimation task and the intracarotid ${ }^{133} \mathrm{Xe}$ washout method. Alteration in sustained attention was correlated with CBF after occlusion using Fisher's exact test.

Results-Deterioration in sustained attention was significantly associated with ipsilateral CBF below $30 \mathrm{ml} / \mathrm{g} / \mathrm{min}$ (Fisher's exact test, $p=0.047$ ). Changes in individual patients' performance on the behavioural task correlated in real time with changes occurring either at the point of occlusion or at a specific threshold of induced hypotension. Sensitivity for our behavioural test in predicting CBF was $75 \%$; specificity $70 \%$.

Conclusions-Monitoring sustained attention during carotid balloon test occlusions seems to be a sensitive means to detect decreases in CBF as measured by ${ }^{133}$ Xe washout. Correlation between higher cerebral function and CBF can allow more detailed investigations of physiological relations between cerebral haemodynamics and brain function in human conditions of cerebral ischaemia.

(F Neurol Neurosurg Psychiatry 1999;66:734-738)

Keywords: cerebral blood flow; carotid occlusion; sustained attention

Acute occlusion of the internal carotid artery (ICA) occurs in various settings, including atherothrombotic stroke, carotid dissection, and carotid endarterectomy. Whereas the consequences of unanticipated ICA occlusion are unpredictable, ranging from no symptoms to devastating hemispheric infarction, patients with inoperable carotid aneurysms or head and neck tumours involving the carotid artery often undergo intentional, temporary ICA occlusion in anticipation of possible carotid sacrifice required for treatment. Such "test occlusions" provide a well controlled environment to investigate the effects of reversible acute hemispheric hypoperfusion on brain function. If the temporary occlusion produces no ill effects for the patient, it is assumed that the collateral circulation from other arterial routes is sufficient for the patient to tolerate permanent occlusion of that carotid.

The standard ICA test occlusion relies on periodic neurological examinations and quantitative cerebral blood flow measurements to monitor the effects of the occlusion. ${ }^{1-4}$ Good clinical measures capable of detecting early alterations of brain function during acute cerebral ischaemia are lacking, however. The extent to which ischaemic neurons remain functional may be different for gross deficits such as hemiparesis compared with higher cerebral functions such as sustained attention. ${ }^{5}$ We developed a simple continuous time estimation task as a quantifiable measure of sustained attention, and showed that the test consistently detected subtle changes in brain function before the emergence of any gross neurological deficit in awake patients undergoing carotid test occlusions. ${ }^{6}$ In the current study, we applied our sustained attention monitoring technique to a series of patients undergoing carotid balloon test occlusions, and simultaneously measured ipsilateral hemispheric cerebral blood flow by the intracarotid ${ }^{133} \mathrm{Xe}$ washout method. We wished to test the hypothesis that alteration in sustained attention would correlate significantly with hemispheric $\mathrm{CBF}$, and provide a more sensitive measure of cerebral ischaemia for ICA test occlusion. This is the first study to our knowledge to test the association between early changes in higher cerebral function and decreased CBF.

Materials and methods

SUBJECTS

Twenty five patients with inoperable pericavernous carotid aneurysms (19 patients) or head and neck tumours (six patients) who underwent test occlusions of the ICA between December 1994 and December 1997 were 


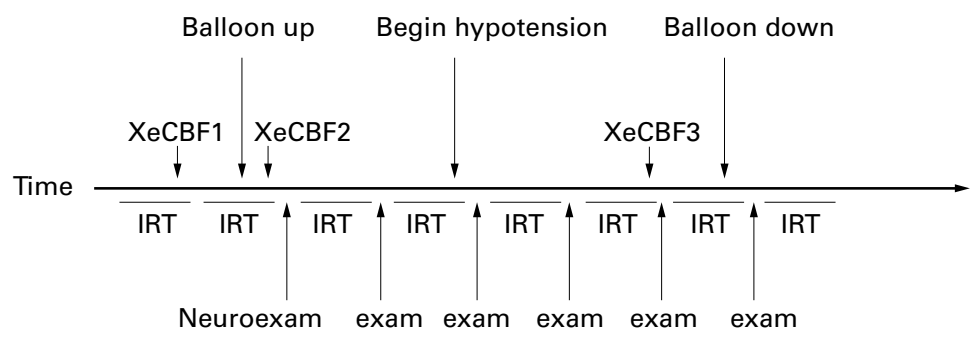

Figure 1 Protocol for ICA balloon test occlusion. Time line indicates timing of balloon inflation and deflation, induction of hypotension, CBF measurements, time estimation (IRT) assessments, and neurological examinations.

studied. There were six men and 19 women with mean age 58.2 years (range 35 to 79 years). Six left ICAs and 19 right ICAs were tested. All patients underwent diagnostic angiograms before test occlusions. Signed, informed consent was obtained for all procedures.

PROCEDURES

Under systemic heparinisation, a 5 French catheter with a balloon tip (OB-5 Meditech ${ }^{\circledR}$ : Boston Scientific Inc, Watertown, MA, USA) was inserted via a 5.5 French femoral artery introducer sheath into the target carotid artery so that the balloon tip was positioned in the mid-cervical portion of the ICA. A distal lumen in the balloon catheter allowed measurement of distal ICA pressures and enabled injection of ${ }^{133} \mathrm{Xe}$ during occlusion. Mean systemic arterial pressure (MAP) was measured with a strain gauge in the femoral introducer sheath. Cortical CBF was determined from intracarotid ${ }^{133} \mathrm{Xe}$ injection, using two cadmium telluride scintillation detectors placed over the lateral frontal and parietal cortex ipsilateral to the carotid occlusion. CBF was calculated with tracer data collected between 20 and 80 seconds after injection, which provided a value weighted toward grey matter. ${ }^{7}$ Arterial blood samples for measurement of $\mathrm{PaCO}_{2}$ and packed cell volume were obtained with each hemispheric CBF determination. Neurological function and performance of the time estimation task (see below) were assessed at the time of baseline CBF measurement. Then, while the patient performed the behavioural task, the balloon was inflated, pressures recorded, and CBF was determined again. As the test occlusion proceeded, standard neurological examinations were done 5 minutes after occlusion, and then every 4-5 minutes between the 3-5 minute blocks of the continuous time estimation procedure (fig 1). The neurological examination, performed by a neurologist, consisted of a standard protocol testing motor and sensory function in the face and limbs, visual fields, verbal fluency and comprehension, and visual extinction to double simultaneous stimuli. If the patient remained clinically stable with initial occlusion, intravenous sodium nitroprusside was then given to induce stages of increasing, graded hypotension to challenge further the adequacy of collaterals. ${ }^{189}$ Nitroprusside was titrated, beginning at a dose of 0.5 $\mu \mathrm{g} / \mathrm{kg} / \mathrm{min}$ and gradually increased up to $1-2$ $\mu \mathrm{g} / \mathrm{kg} / \mathrm{min}$, to achieve a mean arterial pressure
(MAP) of $60 \%-70 \%$ of the patient's preocclusion baseline MAP. Neurological and time estimation task assessments were continued during the hypotensive phase. CBF was recorded again in most patients at the maximum hypotensive stage. The balloon was deflated if either $(a)$ the patient tolerated carotid occlusion for 30 minutes, including hypotensionconsidered to be a "pass" of the test occlusion, or (b) the patient developed dysarthria, aphasia, field cut, or weakness or sensory loss in the contralateral face, arm, or leg during the procedure. Angiography was repeated after balloon deflation to ensure that no embolic arterial occlusions or vasospasm had occurred.

\section{Time estimation task}

Patients were taught a continuous time estimation procedure as a measure of sustained attention, described in detail elsewhere. ${ }^{6} \mathrm{~Pa}-$ tients were instructed to press a computer mouse button, and then to press it again no sooner than 10 seconds but no longer than 13 seconds after the previous response. The hand ipsilateral to the target carotid artery was used to minimise any effect of contralateral hemiparesis on the task performance. A correctly timed response resulted in a tone; mistimed responses produced a different sound. The interresponse time (IRT) clock, measuring the time between consecutive mouse presses, was restarted with every response. The patients were told to make their estimations as precisely as possible to maximise the number of "correct" tones. Each testing block was composed of 20 time estimation responses. The total number of responses made by each patient under each condition varied between 20 and 90 depending on how long they spent in each condition (for example, how long in the occlusion condition before hypotension was begun), but typically patients completed two to three blocks of 20 responses per condition. Patients were trained on the task before the test occlusion until further trials did not improve performance. Performance at that point was interpreted as "baseline." Once in the angiography suite, baseline IRT performance was established with the catheter in place but before balloon inflation. The balloon was then inflated and assessments of performance on the task were obtained during ICA occlusion and under the condition of occlusion plus hypotension as described above.

\section{DATA ANALYSIS}

Fisher's exact test was used to test the strength of association between hemispheric CBF and change in IRT performance. Patients were grouped into those whose CBFs dropped below $30 \mathrm{ml} / 100 \mathrm{~g} / \mathrm{min}$ and those whose CBFs remained at or above $30 \mathrm{ml} / 100 \mathrm{~g} / \mathrm{min}$. This threshold $\mathrm{CBF}$ value represented $2 \mathrm{SD}$ below normal mean blood flow and has been cited as a threshold value in recent investigations. ${ }^{10}{ }^{11}$ CBFs were not known at the time of the IRT testing, so no observer bias could be introduced. The performance measure of interest for the IRT task was variability in precision of time estimation, a behavioural parameter 
Table 1 Patient characteristics, side ICA occluded, IRT variance, CBF, and whether the $C B F$ was measured at hypotension for 25 patients who underwent ICA test occlusion

\begin{tabular}{llllll}
\hline Patient & Age/sex & $\begin{array}{l}\text { ICA } \\
\text { occluded }\end{array}$ & $\begin{array}{l}\text { IRT variance } \\
\text { (baseline, occl) }\end{array}$ & $\begin{array}{l}\text { CBF } \\
\text { (baseline, occl) }\end{array}$ & Hypotension \\
\hline 1 & $60 / \mathrm{M}$ & $\mathrm{L}$ & $2.35,13.3$ & 46,29 & $\mathrm{Y}$ \\
2 & $62 / \mathrm{M}$ & $\mathrm{R}$ & $0.36,0.81$ & 33,32 & $\mathrm{~N}$ \\
3 & $59 / \mathrm{F}$ & $\mathrm{R}$ & $0.97,4.64$ & 34,34 & $\mathrm{~N}$ \\
4 & $78 / \mathrm{F}$ & $\mathrm{R}$ & $0.69,19.5$ & 78,31 & $\mathrm{Y}$ \\
5 & $64 / \mathrm{F}$ & $\mathrm{L}$ & $9.95,14.5$ & 36,31 & $\mathrm{Y}$ \\
6 & $57 / \mathrm{F}$ & $\mathrm{R}$ & $0.32,4.51$ & 49,49 & $\mathrm{Y}$ \\
7 & $48 / \mathrm{F}$ & $\mathrm{R}$ & $2.68,5.12$ & 54,54 & $\mathrm{~N}$ \\
8 & $47 / \mathrm{M}$ & $\mathrm{R}$ & $2.22,2.58$ & 36,39 & $\mathrm{Y}$ \\
9 & $52 / \mathrm{M}$ & $\mathrm{R}$ & $0.16,0.12$ & 31,24 & $\mathrm{Y}$ \\
10 & $61 / \mathrm{F}$ & $\mathrm{R}$ & $4.21,7.48$ & 49,28 & $\mathrm{~N}$ \\
11 & $47 / \mathrm{F}$ & $\mathrm{R}$ & $0.14,0.28$ & 49,42 & $\mathrm{~N}$ \\
12 & $74 / \mathrm{F}$ & $\mathrm{R}$ & $12.3,77.1$ & 37,13 & $\mathrm{Y}$ \\
13 & $66 / \mathrm{F}$ & $\mathrm{R}$ & $9.02,83.1$ & 37,20 & $\mathrm{Y}$ \\
14 & $76 / \mathrm{F}$ & $\mathrm{R}$ & $0.39,0.42$ & 49,33 & $\mathrm{Y}$ \\
15 & $58 / \mathrm{F}$ & $\mathrm{L}$ & $4.14,3.03$ & 56,28 & $\mathrm{Y}$ \\
16 & $41 / \mathrm{F}$ & $\mathrm{R}$ & $0.41,13.2$ & 38,29 & $\mathrm{Y}$ \\
17 & $35 / \mathrm{F}$ & $\mathrm{R}$ & $0.40,3.12$ & 59,43 & $\mathrm{~N}$ \\
18 & $67 / \mathrm{F}$ & $\mathrm{L}$ & $12.1,91.0$ & 37,20 & $\mathrm{Y}$ \\
19 & $63 / \mathrm{F}$ & $\mathrm{R}$ & $1.38,2.34$ & 48,14 & $\mathrm{Y}$ \\
20 & $38 / \mathrm{F}$ & $\mathrm{R}$ & $1.22,0.48$ & 49,43 & \\
21 & $71 / \mathrm{F}$ & $\mathrm{R}$ & $0.46,1.02$ & 53,34 & $M, 28$ \\
22 & $79 / \mathrm{F}$ & $\mathrm{R}$ & $1.34,6.43$ & 54,45 & \\
23 & $36 / \mathrm{F}$ & $\mathrm{L}$ & $1.73,2.50$ & 43,20 & \\
24 & $38 / \mathrm{M}$ & $\mathrm{L}$ & $0.27,4.24$ & 41,27 & \\
25 & $78 / \mathrm{M}$ & $\mathrm{R}$ & $0.29,0.80$ & & \\
\hline & & & & & \\
\hline
\end{tabular}

which is thought to reflect internal clock mechanisms and sustained attention, both of which are required for normal cognitive function. ${ }^{12}$ Variability was represented in this study simply by the variance in timing responses under each condition. As patients' attention waned, their time estimations varied further outside the target of 10 to 13 seconds. For each patient the change in IRT variance from baseline performance to performance under the occlusion condition was measured. For those patients in whom hypotension was induced and there was a CBF measurement at hypotension, the performance under occlusion plus hypotension was used to compare with baseline. Although all patients performed the time estimation task continually throughout the test occlusion, the total number of time estimations made varied from patient to patient. "Baseline" was therefore taken as the final 20 time estimations before occlusion, "occlusion" condition was taken as the first complete block of 20 responses made after occlusion (beginning about 1 to 3 minutes after balloon inflation), and "occlusion plus hypotension condition" as the block of 20 time estimations made at maximum hypotension. A logarithmic transformation was used to reduce skew from outliers. A threshold for change in IRT variance of $\log$ ratio $=0.85$ was chosen to achieve $75 \%$ (top quartile) sensitivity for the task (see results section). Of note, there was a range of abilities on the test at baseline across patients tested. Because the log ratio of the change in IRT variance was used as the performance measure, patients acted as their

Table 2 Sustained attention (IRT) performance by hemispheric $C B F$

\begin{tabular}{llll}
\hline & $\begin{array}{l}C B F<30 \\
\mathrm{ml} / \mathrm{g} / \mathrm{min}\end{array}$ & $\begin{array}{l}\mathrm{CBF} \geqslant 30 \\
\mathrm{ml} / \mathrm{g} / \mathrm{min}\end{array}$ & \\
\hline Ln IRT change $\geqslant 0.85$ & 9 & 4 & 13 \\
Ln IRT change $<0.85$ & 3 & 9 & 12 \\
& 12 & 13 & 25 \\
\hline
\end{tabular}

own controls, "normalising" measurement of perfomance outcome.

\section{Results}

All patients were able to understand the task and perform it according to instructions. Table 1 summarises the patient characteristics, IRT variances, and CBF data for all patients under baseline and occlusion conditions. Of the 25 test occlusions, $\mathrm{CBF}$ measurements were made at hypotension in 15 . Average baseline CBF for all patients was 46 (SD 10.9) $\mathrm{ml} / 100 \mathrm{~g} / \mathrm{min}$. Of the 25, 12 patients had a drop in CBF to below $30 \mathrm{ml} / 100 \mathrm{~g} / \mathrm{min}$ in the occlusion or occlusion plus hypotension condition; 13 remained above $30 \mathrm{ml} / 100 \mathrm{~g} / \mathrm{min}$. Some worsening of IRT performance was seen in all but four patients. Among those whose CBF dropped below 30 $\mathrm{ml} / 100 \mathrm{~g} / \mathrm{min}$, the average IRT variance change was $\log$ ratio 1.52 , representing a 4.5 -fold increase in IRT variance. The average IRT change for those whose CBF remained above $30 \mathrm{ml} / 100 \mathrm{~g} / \mathrm{min}$ was $\log$ ratio 0.93 , representing a 2.5 -fold increase in variance. Taking a $\log$ ratio of 0.85 as a cut off for change in IRT performance achieved a sensitivity of the IRT test of $75 \%$. Specificity for the IRT test at this degree of change was $70 \%$. The association between hemispheric CBF and change in IRT was statistically significant (Fisher's exact test, $\mathrm{p}=0.047$, two tailed, S Plus statistical package) (table 2). The linear correlation between change in IRT and CBF was negative (greater change in IRT correlated with lower CBF), but not statistically significant ( $r=-0.23$, NS).

The standard neurological examination was also administered during the test occlusion. In five patients, arm weakness occurred-in two after occlusion alone and in three after hypotension was induced. Although the specificity was $100 \%$ for this sign (all five with hemiparesis had falls in CBF to below $30 \mathrm{ml} / 100$ $\mathrm{g} / \mathrm{min}$ ), the sensitivity of the standard neurological examination was only $33 \%$ (10 had significant falls in CBF without any sign of weakness). No other neurological sign from the standard examination protocol was seen in any patient. Angiographic images taken after test occlusions showed that no embolic arterial occlusions had occurred. All adverse motor and sustained attention effects reversed when the balloon was deflated. Three patients underwent extracranial-intracranial bypass based on the appearance of a hemiparesis and deterioration in IRT performance during the test occlusion.

Figure 2 illustrates one patient's performance (patient 7) on the IRT time estimation task during test occlusion and under conditions of different pharmacologically induced blood pressures. During this test occlusion, clear alterations in time estimation accuracy were seen, related to changes in systemic blood pressure. A mild right pronator drift appeared at the lowest blood pressure during this test occlusion, then disappeared as the pressure was allowed to increase. 


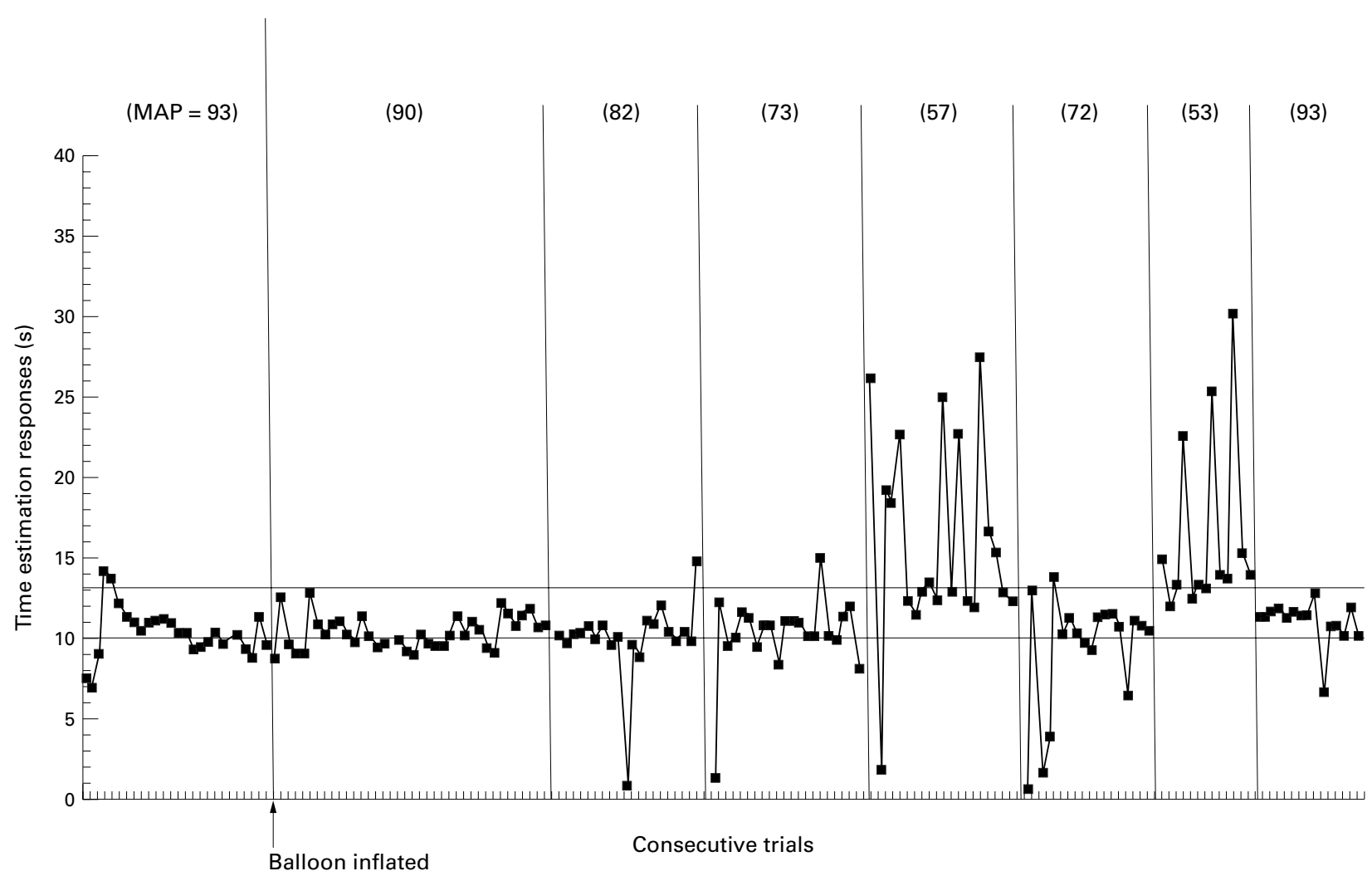

Figure 2 Timing behaviour data for patient 7. Consecutive time estimations plotted during the test occlusion. Timing behaviour remained stable after right ICA occlusion until mean arterial pressure (MAP) was decreased to $57 \mathrm{~mm} \mathrm{Hg}$, at which point time estimations varied widely outside the target interval of 10-13 seconds. Timing behaviour normalised as the pressure was increased, then deteriorated again when the pressure was reduced once again. In the final period, with blood pressure again normal, timing behaviour returned to baseline.

\section{Discussion}

We found a significant association between performance on a sustained time estimation task and level of ipsilateral cerebral blood flow during temporary carotid artery occlusion. Our findings suggest that a simple, quantifiable, and reproducible test of higher cerebral function can detect early changes in hemispheric $\mathrm{CBF}$ during carotid test occlusion with greater sensitivity than the standard neurological examination. Sustained attention may be reversibly altered in this setting, and used to monitor a patient's tolerance of hypoperfusion. In addition to the potential clinical utility of this protocol for ICA test occlusions, to our knowledge this is the first study to quantify an association between real time changes in cerebral blood flow and higher cerebral function in a controlled setting using human subjects.

Our results suggest a strong relation between hemispheric hypoperfusion and altered brain function. Studies of cerebral haemodynamics show that autoregulatory mechanisms work to preserve cerebral metabolic rate of oxygen $\left(\mathrm{CMRO}_{2}\right)$ in the face of falling cerebral perfusion pressure and falling cerebral blood flow. ${ }^{13-15}$ This study did not consider whether sustained attention deteriorated when maximal vasodilation was reached, when oxygen extraction was maximised, or at some other physiological point along a path of reversible ischaemia. By dichotomising our patients into those whose $\mathrm{CBF}$ fell above or below a specific threshold (30 ml/100 g/ min) we obtained a statistically significant correlation with our behavioural performance measure. It might be hypothesised that specific brain regions or a combination of regions must receive a minimum level of blood flow for a patient to maintain good performance on the task, and that below that threshold, the behaviour will begin to fail. The existence of a threshold CBF above which brain function remains normal and below which it deteriorates may be the reason why there did not seem to be a linear correlation of behaviour along a continuum of $\mathrm{CBF}$ values. We chose our $\mathrm{CBF}$ threshold value a priori to be consistent with recent investigations, representing 2 SD below mean $\mathrm{CBF}$ in normal subjects. ${ }^{10}{ }^{11}$ Exact neuronal ischaemic thresholds have not been determined in awake patients, and may differ among individual patients based on age, degree of atherosclerosis, and perhaps most important, on the brain function being measured.

The IRT is a novel means of monitoring brain function in the setting of ICA test occlusions. ${ }^{6}$ Because we did not know in advance how much of a deterioration in time estimation performance would be clinically relevant, we chose a priori a performance threshold that would provide a sensitivity of $75 \%$. This sensitivity was more than twice that of limb weakness as a sign of low CBF in our patients. Although we were left with three test occlusions in which the IRT did not change but the CBFs fell to below $30 \mathrm{ml} / 100 \mathrm{~g} / \mathrm{min}$, the specificity of our IRT test remained at $70 \%$. For those patients in whom sustained attention remained unchanged but the $\mathrm{CBF}$ dropped to 
below $30 \mathrm{ml} / 100 \mathrm{~g} / \mathrm{min}$, it may be that the CBF threshold for altered attention was lower than in other patients. For the four patients in whom there was a significant deterioration in timing behaviour, but the $\mathrm{CBF}$ remained above 30 $\mathrm{ml} / 100 \mathrm{~g} / \mathrm{min}$, it may be that our test was sensitive enough to detect behavioural changes even with minimal decreases in CBF. CBF detector placement and timing of radioactivity count acquisition may also have played a part in these cases.

The anatomical substrate of our time estimation task is not yet known. Both sustained attention and timing are required for the task. Dysfunctional short duration timing behaviour has been correlated in other studies with damage to the cerebellum, ${ }^{16}$ the basal ganglia, ${ }^{17} 18$ the frontal lobes ${ }^{19}$ and, in one patient, to the suprachiasmatic nucleus of the hypothalamus. ${ }^{20}$ Sustained attention has been mapped to paramedian structures such as the anterior and posterior cingulate gyri, and the right prefrontal and superior parietal lobes. ${ }^{21}$ Because we saw both passes and failures of the IRT with both right and left hemisphere, our data may suggest that the test is not hemisphere specific. It may be that sustained attention and timing behaviour are broadly distributed or at least bihemispherically represented. A lack of hemispheric specificity for the task would make the test more useful clinically, as it could then be used with test occlusion of either carotid. We tested far more right ICAs than left ICAs, however, making our numbers too small to test formally for hemispheric laterality. The site of greatest hypoperfusion after occlusion of an ICA will vary according to individual variation in circle of Willis anatomy, but it most often occurs in the "watershed" between the middle and anterior cerebral arteries. Deterioration on our timing task may therefore localise to the high cortical convexity on either hemisphere. It is interesting to note that with the exception of one patient, our behavioural task demonstrated effects of carotid occlusion in the absence of or before proximal arm weakness occurred, a function which has been mapped to this distal field region. ${ }^{22}{ }^{23}$ The one patient who developed arm weakness before the development of deterioration in performance of the IRT test had hypotension induced more precipitously than all others, with the mean arterial pressure dropping from 78 to $50 \mathrm{~mm} \mathrm{Hg}$ in 4 minutes.

We conclude that continuous monitoring of higher cerebral function during carotid balloon test occlusion is not only feasible, but robust and quantifiable. Behavioural monitoring during carotid balloon test occlusion seems to be more sensitive than the standard neurological examination to predict decreases in $\mathrm{CBF}$ as measured by ${ }^{133} \mathrm{Xe}$ washout. A clear correlation between a continuous behavioural task and a physiological measure of cerebral blood flow may provide a basis to predict other haemodynamic, physiological, and behavioural effects in other human conditions of acute cerebral ischaemia.

1 Young WL, Pile-Spellman J. Anesthetic considerations for interventional neuroradiology. Anesthesiology 1994;80:42756.

2 Larson JJ, Tew JM, Tomsick TA, et al. Treatment of aneurysms of the internal carotid artery by intravascular balloon occlusion: long-term follow-up of 58 patients. Neurosurgery 1995;36;23-30

3 Vazquez Añon V, Aymard A, Gobin YP, et al. Balloon occlusion of the internal carotid artery in 40 cases of giant intracavernous aneurysm: technical aspects, cerebral monitoring, and results. Neuroradiology 1992;34:245-51.

4 Witt J-P, Yonas H, Jungreis C. Cerebral blood flow response pattern during balloon test occlusion of the internal carotid artery. AfNR Am 7 Neuroradiol 1994;15:847-57.

$5 \mathrm{Mohr}$ JP. Some clinical aspects of acute stroke. Excellence in clinical stroke award lecture. Stroke 1997;28:1835-9.

6 Lazar RM, Marshall RS, Pile-Spellman H, et al. Continuous time estimation as a behavioral index of human cerebral ischaemia during temporary occlusion of the internal carotid artery. $\mathcal{F}$ Neurol Neurosurg Psychiatry 1996;60:55963.

7 Young WL, Prohovnik I, Schroeder T, et al. Intraoperative ${ }^{33} \mathrm{Xe}$ cerebral blood flow measurements by intravenous versus intracarotid methods. Anesthesiology 1990;73:637-43.

8 Tanaka F, Nishizawa S, Yonekura Y, et al. Changes in cerebral blood flow induced by balloon test occlusion of the internal carotid artery under hypotension. Eur $\mathcal{F}$ Nucl Med 1995;22:1268-73.

9 Standard SC, Ahuja A, Guterman LR, et al. Balloon test occlusion of the internal carotid artery with hypotensive challenge. AfNR Am f Neuroradiol 1995;16:1453-8.

10 Brunberg JA, Frey KA, Horton JA, et al. $\left[{ }^{15} \mathrm{O}\right] \mathrm{H}_{2} \mathrm{O}$ positron emission tomography determination of cerebral blood flow during balloon test occlusion of the internal carotid artery. AfNR Am f Neuroradiol 1994;15:725-32.

11 Linskey ME, Jungreis CA, Yonas H, et al. Stroke risk after abrupt internal carotid artery sacrifice: accuracy of preoperative assessment with balloon test occlusion and stable xenon-enhanced CT. AfNR Am f Neuroradiol 1994;15: 829-43.

12 Gibbon J, Malapani C, Dale CL, et al. Toward a neurobiology of temporal cognition: advances and challenges. Curr Opin Neurobiol 1997;7:170-84.

3 Powers WJ. Cerebral hemodynamics in ischemic cerebrovascular disease. Ann Neurol 1991;29:231-40.

14 Powers WJ, Grubb RL, Raichle ME. Physiological responses to focal cerebral ischemia in humans. Ann Neurol 1984;16:546-52

15 Sette G, Baron JC, Mazoyer B, et al. Local brain haemodynamics and oxygen metabolism in cerebrovascular disease: positron emission tomography. Brain 1989;112:931-51.

16 Ivry RB, Keele SW. Timing functions of the cerebellum. 7 Cogn Neurosci 1989;1:136-52.

17 Wing A, Keele S, Margolin D. Motor disorder and the timing of repetitive movements. Ann NY Acad Sci 1984;423: ing of reper.

18 O'Boyle DJ, Freeman JS, Cody FWJ. The accuracy and precision of timing of self-paced, repetitive movements in subjects with Parkinson's disease. Brain 1996;119:51-70.

19 Nichelli P, Clark K, Hollnagel C, et al. Duration and processing after frontal lobe lesions. Ann NY Acad Sci 1995;769:183-90.

20 Cohen RA, Barnes HJ, Jenkins M, et al. Disruption of shortduration timing associated with damage to the suprachias1533-9.

21 Pardo JV, Fox PT, Raichle ME. Localization of a human system for sustained attention by positron emission tomography. Nature 1991;349:61-4.

22 Colebatch JG, Deiber MP, Passingham RE, et al. Regional cerebral blood flow during voluntary arm and hand movements in human subjects. $\mathcal{F}$ Neurophysiol 1991;65:1392401 .

23 Rao SM, Binder JR, Hammeke TA, et al. Somatotopic mapping of the human primary motor cortex with functional magnetic resonance imaging. Neurology 1995;45:919-24. 